\title{
Update on Lung Cancer
}

Nicole Bouchard MD, FRCPC; Jason Scott Agulnik MD, CM, BSc

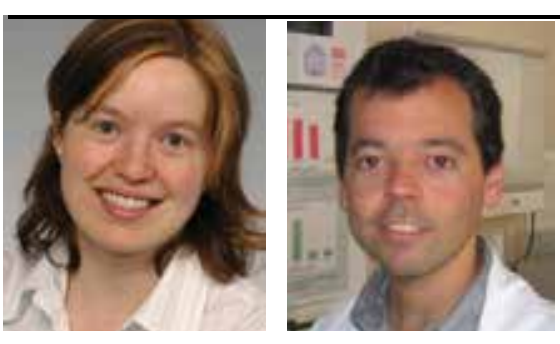

\begin{abstract}
About the Authors
Nicole Bouchard is a Respirologist and Associate Professor of Medicine at the Université de Sherbrooke in Quebec and a member of the Respiratory Service of the Department of Medicine at the Centre Hospitalier Universitaire de Sherbrooke. Jason Scott Agulnik is a Respirologist and Assistant Professor in the Division of Pulmonary Diseases at the Department of Medicine at Sir Mortimer B. Davis-Jewish General Hospital. He is also an Assistant Professor in the Department of Oncology at McGill University in Montreal, QC, Canada. Correspondence may be directed to nicole.bouchard@usherbrooke.ca.
\end{abstract}

\section{Summary}

Investigation and treatment of lung cancer has changed dramatically since the last articles in this journal in 2008. These changes include a new study on lung cancer staging, a new tumour, node, metastasis (TNM) classification, linear endoscopic ultrasound as a first-line test for mediastinal staging, investigation and follow-up of ground glass and mixed-lung nodules, radiosurgery for inoperable patients with localized lung tumours, and molecular tests and targeted therapies for advanced non-small cell lung cancer.

\section{Résumé}

L'investigation et le traitement du cancer du poumon ont énormément évolué depuis les derniers articles sur le sujet parus dans cette revue en 2008. Ont contribué à cette évolution une nouvelle étude sur la stadification du cancer du poumon, une nouvelle classification TNM, l'échoendoscopie devenue l'examen de première intention dans la stadification médiastinale, l'investigation et le suivi du nodule en verre dépoli et du nodule mixte, la radiochirurgie chez le patient inopérable dont les tumeurs sont localisées et les analyses moléculaires et les traitements ciblés dans le cancer du poumon non à petites cellules de stade avancé. 


\section{Screening}

According to the 2014 statistics for Canada, lung cancer is still the leading cause of cancer-related deaths and has the second highest incidence, with approximately 26,000 new diagnoses. A major American study (National Lung Screening Trial ${ }^{1}$ [NLST]) that included more than 50,000 patients was published in 2011. It showed a decrease in lung cancer mortality of $20 \%$ and all-cause mortality of $6.7 \%$. A total of 320 patients had to be screened to save one death by lung cancer. A low-dose computerized axial tomography (CT) scan was done yearly for three consecutive years and compared to a chest X-ray only. Inclusion criteria were age between 55 and 74 years and smoking history of at least 30 pack-years (number of packs of cigarettes smoked per day divided by the number of years the person has smoked). Patients were either still smoking or had stopped in the last 15 years. Since that publication, many medical associations (American Cancer Society of Clinical Oncology [ASC], Cancer Care Ontario, and US Preventive Services Task Force [USPSTF]) have recommended lung cancer screening. However, lung cancer screening is still not a standard of care in the country, due to remaining questions regarding false positives, frequency or length of screening after three years, and concerns about the cumulative risk of radiation. There is also a risk of over-diagnosis related to a subtype of adenocarcinomas, which used to be called bronchioloalveolar carcinoma.

If screening is proposed to a given patient, benefits and risks should be discussed before asking for a computed tomography (CT) scan. The Dutch-Belgian Randomized Lung Cancer Screening Trial (NELSON) being done in Europe will help to answer these questions, and its results should be available in 2016 .

\section{Diagnosis and Staging \\ Histological Classification and Staging}

The seventh tumour, node, metastasis (TNM) classification on lung cancer was published in $2009^{2}$ due to the prognostic impact on survival (Table 1). It includes both small cell and non- small cell lung cancers. Small cell lung cancers are no longer classified as limited or extensive.

A stage I lung cancer is a tumour less than $5 \mathrm{~cm}$ without metastasis in lymph nodes. A stage II is a tumour larger than 5 $\mathrm{cm}$ or that invades certain adjacent structures, or a small tumour with metastatic lymph nodes either in the hilum, interlobar region, or lung. A stage III is a tumour that invades a central organ (such as the heart, big vessels, trachea, or oesophagus) or metastatic lymph nodes in the mediastinum, scalene, or supraclavicular region. A stage IV invades pleura or pericardial
Table 1. Descriptors, Proposed T and M Categories, and Proposed Stage Groupings

\begin{tabular}{|c|c|c|c|c|c|}
\hline $\begin{array}{l}\text { Sixth Edition } \\
\text { T/M Descriptor }\end{array}$ & $\mathrm{T} / \mathrm{M}$ & NO & N1 & N2 & N3 \\
\hline $\mathrm{T} 1(<2 \mathrm{~cm})$ & T1a & IA & IIA & IIIA & IIIB \\
\hline $\mathrm{T} 1(>2-3 \mathrm{~cm})$ & $\mathrm{T} 1 \mathrm{~b}$ & IA & $\| A$ & IIIA & IIIB \\
\hline $\mathrm{T} 2(<5 \mathrm{~cm})$ & T1a & IB & IIA & IIIA & IIIB \\
\hline $\mathrm{T} 2(>5-7 \mathrm{~cm})$ & $\mathrm{T} 1 \mathrm{~b}$ & IIA & IIB & IIIA & IIIB \\
\hline $\mathrm{T} 2(>7 \mathrm{~cm})$ & T3 & IIB & IIIA & IIIA & IIIB \\
\hline T3 invasion & & IIB & IIIA & IIIA & IIIB \\
\hline T4 (same lobe nodules) & T4 & IIB & IIIA & IIIA & IIIB \\
\hline T4 (extension) & & IIIA & IIIA & IIIB & IIIB \\
\hline M1 (ipsilateral lung) & & IIIA & IIIA & IIIB & IIIB \\
\hline T4 (pleural effusion) & M1a & IV & IV & IV & IV \\
\hline M1 (contralateral lung) & & IV & IV & IV & IV \\
\hline M1 (distant) & M1b & IV & IV & IV & IV \\
\hline
\end{tabular}

Journal of Thoracic Oncology, 2011, Vol.6 No.2, p.246 Reprinted with permission

Table 2. IASLC/ATS/ERS Classification of Lung Adenocarcinoma in Resection Specimens

\section{Preinvasive lesions}

Atypical adenomatous hyperplasia

Adenocarcinoma in situ ( $<3 \mathrm{~cm}$ formerly $\mathrm{BAC})$

Nonmucinous

Mucinous

Mixed mucinous/nonmucinous

Minimally invasive adenocarcinoma $(<3 \mathrm{~cm}$ lepidic predominant tumor with $<5 \mathrm{~mm}$ invasion)

Nonmucinous

Mucinous

Mixed mucinous/nonmucinous

Invasive adenocarcinoma

Lepidic predominant (formerly nonmucinous BAC pattern, with $>5 \mathrm{~mm}$ invasion)

Acinar predominant

Papillary predominant

Micropapillary predominant

Solid predominant with mucin production

Variants of invasive adenocarcinoma

Invasive mucinous adenocarcinoma (formerly mucinous BAC)

Colloid

Fetal (low and high grade)

Enteric

BAC, bronchioloalveolar carcinoma; IASLC, International Association for the Study of Lung Cancer; ATS, American Thoracic Society; ERS, European Respiratory Society. 
effusion or metastases in the other lung or distal organs. Useful resources for information include the staging lung cancer website $^{3}$ (Figure 1) and the IASLC Staging Atlas application. ${ }^{4}$

In 2011, a new adenocarcinoma classification was published. ${ }^{5}$ The term "bronchioalveolar carcinoma" should not be used anymore (Table 2). Non-mucinous bronchioloalveolar carcinomas are now classified as being either in situ, minimally invasive, or lepidic predominant, based on their size and invasive component. They usually are ground glass lesions on CT scan and have an excellent prognosis after curative surgery. Mucinous bronchioloalveolar carcinomas should now be called invasive mucinous adenocarcinomas and they are more often associated with consolidations and are multifocal/ multilobar.

\begin{tabular}{|c|c|c|}
\hline Noguchi 1995 & IASLC/ATS/ERS 2011 & Main CT features \\
\hline & AAH & GGN \\
\hline A, localized BAC & \multirow[b]{2}{*}{ AIS } & GGN \\
\hline $\begin{array}{l}\text { B, localized BAC with } \\
\text { alveolar collapse }\end{array}$ & & GGN/PSN \\
\hline \multirow{3}{*}{$\begin{array}{l}\text { C, localized BAC with active } \\
\text { fibroblastic proliferation }\end{array}$} & MIA & GGN/PSN \\
\hline & $\begin{array}{l}\text { Lepidic predominant } \\
\text { adenocarcinoma } \\
\text { (nonmucinous) }\end{array}$ & $\begin{array}{l}\text { PSN } \\
\text { SN }\end{array}$ \\
\hline & $\begin{array}{l}\text { Invasive mucinous } \\
\text { adenocarcinoma }\end{array}$ & $\begin{array}{l}\text { PSN with } \uparrow \uparrow \text { solid } \\
\text { component } \\
\text { SN } \\
\text { Consolidation }\end{array}$ \\
\hline $\begin{array}{l}D=\text { poorly differentiated } \\
E=\text { tubular } \\
F=\text { papillary tumor }\end{array}$ & $\begin{array}{l}\text { Invasive adenocarcinoma, } \\
\text { classified by the } \\
\text { predominant subtype }\end{array}$ & $\begin{array}{l}\text { SN or PSN with } \uparrow \uparrow \\
\text { solid component }\end{array}$ \\
\hline
\end{tabular}

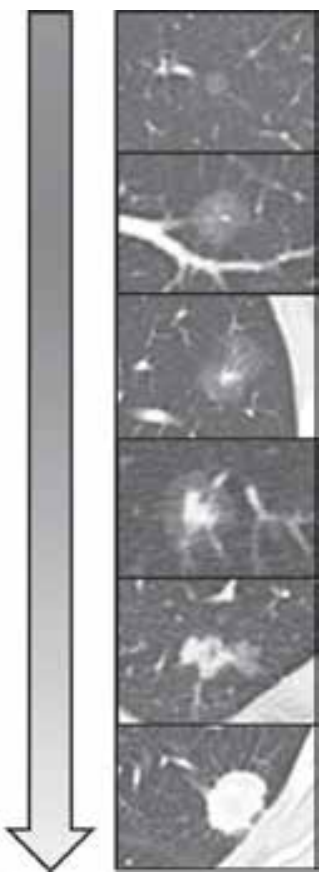

Figure 1. Correlation of the new IASLC/ATS/ERS lung adenocarcinoma classification with the Noguchi classification and the corresponding most common computed tomography features of each subtype of adenocarcinoma. The arrow indicates increased solid attenuation and decreased prognosis as the lesions become more invasive on histology. AAH, atypical adenomatous hyperplasia; AIS, adenocarcinoma in situ; ATS, American Thoracic Society; BAC, bronchioloalveolar carcinoma; CT, computed tomography; ERS, European Respiratory Society; GGN, ground-glass nodule; IASLC, International Association for the Study of Lung Cancer; MIA, minimally invasive adenocarcinoma; PSN, part-solid nodule; SN, solid nodule.

Subsolid pulmonary nodules: imaging evaluation and strategic management. Copyright: www.co-pulmonarymedicine.com, 2012, Vol.18, No. 4, p.307. Reprinted with permission.

In 2013, new recommendations were developed regarding investigation and follow-up of subsolid lesions ${ }^{6}$ (subsolid lesions include both ground glass and partially solid lesions). They are generally more grey than white on CT scan images. Partially solid nodules include a ground glass part and a solid part (which means a grey zone and a white zone; this white zone is usually the invasive part, which is solid). These subsolid lesions have a slower growth than a lung cancer with a solid nodule and have a better prognosis. They often correlate with an adenocarcinoma diagnosis on the pathology report. Their investigation will be discussed in the treatment part of this article.

\section{Investigation}

A chest CT scan with intravenous contrast should be prescribed for every patient. ${ }^{7}$ If a curative treatment is considered (stages I to III), a positron emission tomography (PET) scan should be ordered, if available. Otherwise, a chest CT scan up to the adrenal glands and a bone scintigraphy should be prescribed to complete extrathoracic staging. For small cell lung cancer, a PET scan could be ordered for stage I to III, but data are more controversial. A PET scan does not adequately evaluate brain or distal limbs. Brain staging (CT scan or, preferably, a magnetic resonance imaging) should be done if there are neurologic symptoms and for every small cell lung cancer. It 
should be considered for non-metastatic non-small cell lung cancer, especially for stage III.

For non-small cell lung cancer, the most advanced histological stage should be determined to increase the patient's chances of being cured, unless there are significant risks with the procedure. This is especially important if there is only one site that is suspect of metastases, or if there is no extensive infiltration of the mediastinum. However, for small cell lung cancer, the less invasive test will be done. The whole investigation period should overlap and not be done in a sequential order, to shorten the time of the investigation.

Many studies have confirmed that endobronchial ultrasound (EBUS) combined or not with esophageal ultrasound (EUS) is the first choice to stage hilar or mediastinal lymph nodes that are increased in size on CT scan (smallest diameter at least $1 \mathrm{~cm}$ ) and/or that are positive on PET scan. If the tumour is central, a mediastinal staging should also be done. If clinical suspicion of lymph node metastasis remains after a negative ultrasound ponction, a surgical staging should be done (mediastinoscopy, mediastinotomy, or thoracoscopy).

If there is a mediastinal metastasis, usual bronchoscopy, or transthoracic, biopsy of the lung lesion is not indicated. EBUS +/- EUS will simultaneously provide the diagnosis and establish the stage.

Solid nodules larger than $8 \mathrm{~mm}$ should be considered neoplastic until proven otherwise. Bronchoscopy and transthoracic biopsy (TTB) have high false-negative results. For central lesions, bronchoscopy is diagnostic in about $90 \%$ of cases, but only in $20 \%$ of peripheral lesions. Regarding TTB, the falsenegative rate is $20 \%$. One negative TTB should not exclude a lung cancer diagnosis if the clinical suspicion remains high. Two choices are then offered, either a second TTB or a diagnostic thoracic surgery. Radial EBUS is a new procedure allowing better sampling of a lung nodule than conventional bronchoscopy. The sample is taken under direct ultrasound guidance. This technique is still not widely available and will not replace transthoracic biopsy because the nodule can be too far to be reached with a radial EBUS. Being able to see a bronchus near the nodule improves the diagnostic yield of radial EBUS, with a sensitivity of $80 \%$.

If lung nodules are smaller than $8 \mathrm{~mm}$, they should be called micronodules and be followed by chest CT scan according to cancer risk and their size. Fleischner Society guidelines $^{8}$ are useful to decide the length between follow-up CT scans, to a total follow-up duration of two years. Subsolid nodules (ground glass or mixed solid and ground glass) should be followed in a different way. Guidelines are not standardized for these lesions. Generally, it is important to remember that lung cancer risk is higher for mixed nodules and that annual CT scan follow-up should be longer (3-5 years) than for solid nodules, in order to be more confident about their benign pattern. Also, a first CT scan should be done after three months to know if the lesion has disappeared, because this is more common with subsolid lesions.

If a subsolid nodule increases in size or develops a solid component, a diagnostic procedure must be done. If nodules are larger than $10 \mathrm{~mm}$, and particularly $15 \mathrm{~mm}$, then a diagnostic procedure is also indicated. The size of the solid component ( $5 \mathrm{~mm}$ or more) of a mixed nodule should also be considered in the decision to further investigate the lesion. One must remember that the rate of false negative PET scans and transthoracic biopsies is greater in subsolid nodules than it is in solid nodules.

Because of the impact on therapies for stage IV non-small cell cancers, the histology (adenocarcinoma, squamous cell carcinoma) should be analyzed using immunohistochemistry, if possible. Generally, two markers are used. These markers are TTF1 and p63 (or p40), respectively, markers for adenocarcinoma and squamous cell cancer. There should be enough tissue for molecular tests to be done ulteriorly if the diagnosis is an adenocarcinoma. A search for mutations in the epidermal growth factor receptor (EGFR) gene and rearrangement of the anaplastic lymphoma kinase (ALK) gene is requested for adenocarcinoma tumours. ${ }^{9}$ This will be further discussed in the section on therapy.

For pleural effusions in which cancer is suspected, a pleural tap is often obtained. A chest ultrasound will increase the diagnostic yield and decrease the frequency of complications such as pneumothoraces. Chest ultrasound is recommended to localize the site for thoracocentesis. If two thoracocenteses are negative and the clinical suspicion of cancer remains high, then a pleural biopsy is recommended.

Before considering a lung resection or a treatment by radiation therapy, pulmonary function tests should be obtained, including spirometry and a measure of the lung diffusion capacity. The forced expiratory volume in the first second FEV1 and the lung diffusion capacity should be calculated based on projected postoperative predicted values, according to the percentage of lung that is to be resected. If the predicted postoperative FEV1 and lung diffusion capacity are greater than $60 \%$, then no further pulmonary function testing is required. If not, then a qualitative lung perfusion scan and/ or a cardiorespiratory exercise test should be obtained..$^{10}$ 


\section{Treatment of Non-Small Cell Carcinomas Stages I and II}

Surgical treatment remains the first choice. A procedure with thoracoscopy is preferable for stage I cancers. The lobectomy remains the standard choice with respect to less invasive surgery. Among less invasive surgeries, a segmentectomy is a preferred option with respect to a wedge resection. These options are considered if the pulmonary function tests are prohibitively low or in the presence of significant comorbid conditions. A pneumonectomy is performed only if, from an oncological perspective, a smaller sleeve resection is not possible. For stage II lung cancer with lymph node invasion in a patient with a good performance status, adjuvant platinumbased chemotherapy is recommended over four cycles. Similar recommendations are offered for stage IIIA (N2 node) that is diagnosed postoperatively when the resected tissue is analyzed. Adjuvant radiotherapy is recommended only if the resection is incomplete.

If the patient cannot tolerate surgery, then stereotactic body radiation therapy (SBRT) is recommended for tumours less than $5 \mathrm{~cm}$ in patients with stage I cancer without suspicious lymph nodes. ${ }^{11}$ This therapy consists of high-dose radiation therapy provided in several fractions. The therapy is of a shorter duration (3-5 days) than conventional radiation therapy (30 days). Current studies suggest that local control is excellent with a $90 \%$ success rate three years after the procedure. No phase III clinical trials compare surgical therapy to this new radiation therapy, since most of these studies failed due to lack of recruitment. It will be difficult to obtain a clear comparison between these two therapeutic modalities, particularly since the pathological staging is not done in patients undergoing SBRT, and several patients are not willing to be subjected to the short-term morbidity and mortality associated with surgical therapy. The current indication of SBRT is therefore mainly in patients who have poor lung function tests and other significant comorbid conditions that preclude a surgical resection. If SBRT is not possible, then conventional radiation therapy is recommended, but the results are clearly inferior to surgical resection or SBRT.

\section{Stage III}

The treatment of stage III non-small cell lung carcinomas is more controversial. For most of these patients, a combination of chemotherapy using a platinum-based medication and radiation therapy is recommended if the performance status is good (ECOG 0 or 1 ) and if there is no significant weight loss during treatment (less than 5-10\% weight loss over the last few months). The dose of radiation therapy is approximately
60 Gy. Concomitant chemotherapy and radiation therapy are superior to sequential therapies (chemotherapy followed by radiation therapy). If the patient is too ill, then palliative radiation therapy alone is recommended.

Certain patients will require a neoadjuvant treatment (chemotherapy combined with or without radiation therapy) if the mediastinal disease is limited to a single lymph node in the ipsilateral para-tracheal (N2) region, and this therapy is followed by surgical resection. This approach is controversial, as it has not been demonstrated to be superior to concomitant chemotherapy and radiation therapy.

For patients with stages I or II disease who have undergone lung resection and in whom the postoperative analyses reveal that it is a stage IIIA (N2 lymph node involvement) disease, adjuvant chemotherapy is recommended. Adjuvant radiation therapy is only recommended in patients in whom the lung resection is incomplete. For certain selected cases, if the risk of local recurrence is high, then adjuvant radiation therapy after adjuvant chemotherapy can be considered to decrease the risk of local recurrence.

\section{Stage IV}

Palliative chemotherapy is recommended in patients with a good performance status $(0,1$, or even 2$)$. The first line of therapy depends on the histology and on the results of molecular test for adenocarcinomas. A total of three lines of chemotherapy have been shown to be beneficial for the survival of patients and their quality of life.

For the first line of chemotherapy, survival benefits are approximately 3-4 months when a combination of platinumbased agents (cisplatin or carboplatin) are used with a second agent, such as gemcitabine, paclitaxel, or pemetrexed. Carboplatin is associated with fewer adverse events, such as renal insufficiency, neuropathy, nausea, and vomiting. This treatment is provided over four cycles and sometimes up to six cycles.

If the lung cancer is not a squamous cell type, then pemetrexed can be used, since it is more effective. Pemetrexed is associated with clinical deterioration in patients with squamous cell carcinomas. This agent is contraindicated in patients with squamous cell carcinomas, as is bevacizumab (a monoclonal antibody against vascular endothelial growth factor that is given in combination with chemotherapy). Bevacizumab is rarely prescribed for patients with lung cancer because of its poor cost/benefit ratio.

If the tumour is an adenocarcinoma with an EGFR positive mutation (particularly exons 19 and 21), then a tyrosine kinase inhibitor (gefitinib, erlotinib, afatinib) will be prescribed 
because of the superior efficacy quality-of-life and adverse effects profile. These tumours also generally have a better prognosis. Such tumours are more common in patients who have not smoked cigarettes and who are of Asian origin. If the tumour is an adenocarcinoma with an ALK rearrangement, then an ALK tyrosine kinase inhibitor (crizotinib) will provide superior efficacy and a better quality of life. This is a major change in clinical practice based on targeted therapy. This treatment is an oral medication in contrast to conventional chemotherapy, which is usually intravenous. Furthermore, there is no febrile neutropenia associated with these agents. In the coming years, it is likely that many other targeted therapies will be available for adenocarcinomas, and similar studies are being initiated for squamous cell carcinomas. ${ }^{12}$

Chemotherapy maintenance therapy is possible if there is no evidence of progression after the first line of therapy in patients with a good performance status $(0,1)$. This therapy is continued as long as there is no evidence of progression and the adverse effects are well tolerated. Survival benefits are observed over 1-5 months, according to the histological type and the chemotherapy agent used (pemetrexed, erlotinib).

The second line of therapy includes docetaxel or pemetrexed (if not previously prescribed). These agents provide approximately two months of survival benefit. Erlotinib is also used as a second-line therapy, particularly for patients who are not good candidates for the other second-line therapies. In the third-line therapy, only erlotinib has proven to have some survival benefits.

\section{Prognosis}

Five-year survival depends mainly on the stage of the lung carcinoma (Table 3). The general five-year survival rate is approximately $17 \%$.

Table 3. Prognosis of Non-Small Cell Carcinomas

\begin{tabular}{|l|l|}
\hline Stage & 5 -year survival \\
\hline IA & $73 \%$ \\
\hline IB & $58 \%$ \\
\hline IIA & $46 \%$ \\
\hline IIB & $35 \%$ \\
\hline IIIA & $19-24 \%$ \\
\hline IIIB & $7-9 \%$ \\
\hline IV & $2 \%$ \\
\hline
\end{tabular}

Copyright by Journal of Thoracic Oncology, 2007, Vol. 2, No. 8, p. 709.

Reprinted with permission

\section{Small Cell Carcinoma Therapy}

For stages I to III small cell carcinomas (previous limited stage), chemotherapy based on cisplatin-etoposide associated with radiation therapy is recommended in concomitant therapy. Four cycles of chemotherapy are given. Radiation therapy can be provided according to various protocols, with a total radiation of 40-60 Gray (Gy). A sequential treatment of chemotherapy followed by radiation therapy is prescribed if there are contraindications to concomitant therapy. Very rarely is small cell carcinoma removed surgically, and this is only done if the tumour is of a small size and there is no evidence of lymph node involvement or metastatic disease. Stage IV small cell carcinomas require chemotherapy with a regimen based on platinum and etoposide over four to six cycles.

Cerebral prophylaxis with the radiation therapy is prescribed if there is a complete therapeutic response in the limited stages. At the 2014 American Society of Clinical Oncology Conference (ASCO), evidence was provided demonstrating there is no survival advantage for extensive stages in which cerebral prophylaxis is added, based on the initial imaging of the brain with magnetic resonance. ${ }^{13}$

If recurrence occurs, then a therapy using topotecan or a combination of cyclophosphamide, doxorubicine, vincristine (CAV) can be offered. The response rate is generally poor. If recurrence occurs more than six months after the end of the treatment, then a platinum-etoposide treatment is usually prescribed once again.

\section{Follow-Up}

No standard follow-up has been demonstrated as being beneficial for patient survival. In general, patients will be followed up every 3-6 months for the first 2 years and every 6-12 months for the next 3-5 years. Annual follow-ups will be done afterwards. Routine imaging involves chest X-ray or CT scan. Chest CT scans are usually not obtained more than once a year.

\section{Future Trends}

The coming years will see a more extensive use of endoscopic ultrasound, stereotactic body radiation therapy, and molecular therapy. At ASCO conference 2015, exciting results from trials on immunotherapy have been presented, especially regarding nivolumab in second line therapy for advanced squamous cell carcinoma. 


\section{Conflict of Interest}

The senior author has made several presentations on lung cancer (new TNM classification, endobronchial ultrasound, new therapies for metastatic stages, lung cancer screening) and received honoraria from the pharmaceutical industry for some of these presentations.

\section{References}

1. The National Lung Screening Trial Research Team. Reduced lung-cancer mortality with low-dose computed tomographic screening. N Engl J Med 2011;365:395-409.

2. Detterbeck FC, Boffa DJ, Tanoue LT. The new lung cancer staging system. Chest 2009;136:260-71.

3. Staging Lung Cancer [website]. Available at: www.staginglungcancer.org. Accessed September 23, 2014.

4. IASLC Staging Atlas application. Accessed September 23, 2014.

5. Travis WK, Brambilla E, Noguchi M, et al. International Association for the Study of Lung Cancer/American Thoracic Society/European Respiratory Society - International multidisciplinary classification of lung adenocarcinoma. J Thorac Oncol 2011;6:244-85.
6. Naidich DP, Bankier AA, MacMahon H, et al. Recommendations for the management of subsolid pulmonary nodules detected at CT: a statement from the Fleischner Society. Radiology 2013;266:304-17.

7. Detterbeck FC, Zelman Lewis S, Diekemper R, et al. Executive summary: diagnosis and management of lung cancer, 3rd ed. American College of Chest Physicians. Chest 2013;143:7S-37S.

8. MacMahon H, Austin JHM, Gamsu G, et al. Guidelines for management of small pulmonary nodules detected on CT scans: a statement from the Fleischner Society. Radiology 2005;237:395-400.

9. Lindeman NI, Cagle PT, Beasley MB, et al. Molecular testing guideline for selection of lung cancer patients for EGFR and ALK tyrosine kinase inhibitors. J Thorac Oncol 2013;8:823-59.

10. Lim E, Baldwin D, Beckles M, et al. Guidelines on the radical management of patients with lung cancer. Thorax 2010;65(Suppl III):iii1-iii27.

11. Timmerman R, Paulus R, Galvin J, et al. Stereotactic body radiation therapy for inoperable early stage lung cancer. JAMA 2010;303:1070-6.

12. Kris MG, Johnson BE, Berry LD, et al. Using multiplexed assays of oncogenic drivers in lung cancers to select targeted drugs. JAMA 2014;311:1998-2006.

13. Seto T, Takahashi T, Yamanaka T, et al. Prophylactic cranial irradiation (PCI) has a detrimental effect on the overall survival (OS) of patients (pts) with extensive disease small cell lung cancer (ED-SCLC): results of a Japanese randomized phase III trial. J Clin Oncol 32:5s;2014(Suppl abstr 7503).

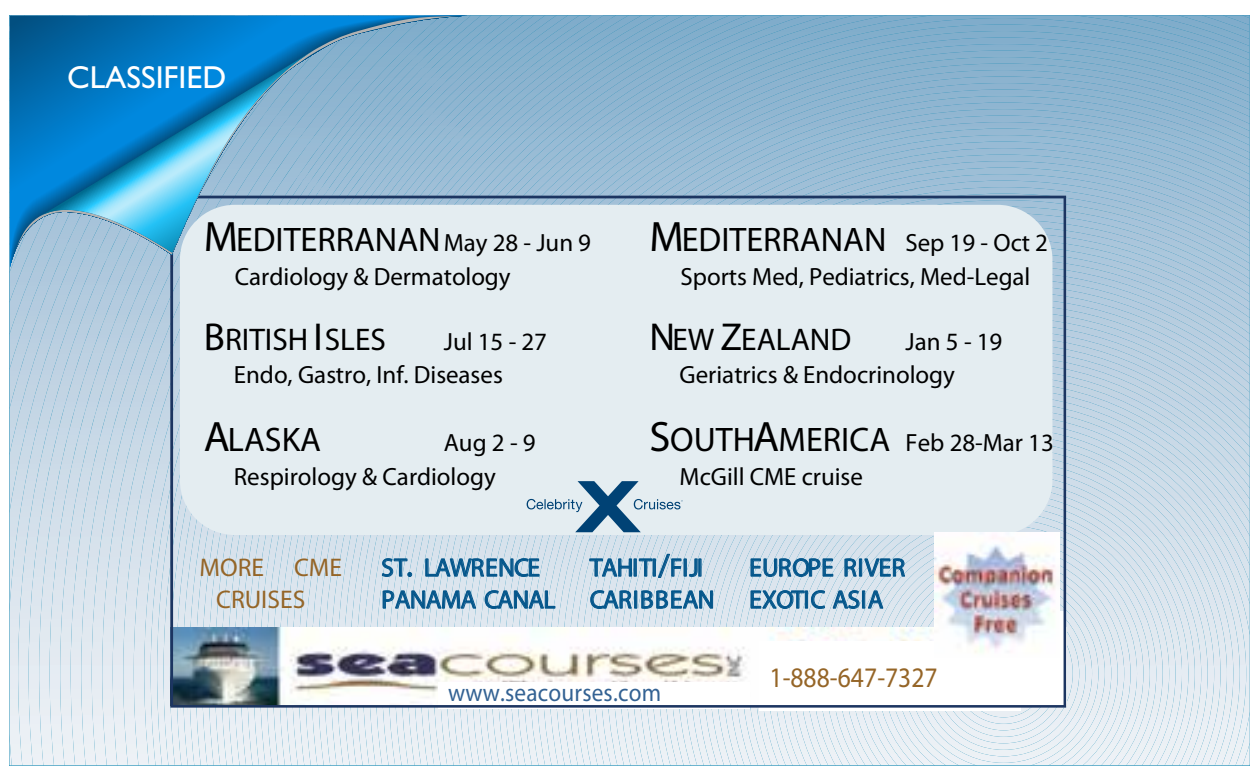

